K.-P. CHENG

KODAI MATH. J.

2 (1979), 362-370

\title{
THE HORIZONTAL HOLONOMY GROUP OF A FIBRE BUNDLE SPACE
}

\author{
By Koun-Ping Cheng
}

\section{$\S 1$. Introduction.}

On a differentiable manifold $M$, the parallel translation of a vector along a curve $C$ has been studied in many papers and books. A topological group $H(M)$ was assigned to this manifold $M$. And we call $H(M)$ the linear holonomy group of $M$. Nijenhuis, in his paper ([1]), found out that the Lie algebra of the restricted holonomy group $H^{0}(M)$ of $H(M)$ is formed by the curvature tensor of $M$. On the other hand, if we consider the frame bundle $B(M)$ as a principle fibre bundle over $M$, then the Nijenhuis's theorem can be restated as follows: The holonomy Lie algebra of $H^{0}(M)$ is generated by the curvature form $\Omega$ of $B(M)$ ([2]).

We know that a principle fibre bundle is only a special case of a fibre bundle space. Hence, the ideal of the linear holonomy group can be extended to the fibre bundle space. Actually, if we consider any fibre bundle space $(\tilde{M}, M, \pi)$ such that in $\tilde{M}$, there is a 1 -form $\omega$ and $\omega$ can determine the horizontal vectors of $\tilde{M}$, then $\tilde{M}$ can have a horizontal holonomy group $H l(\tilde{M})$ (see section 2) associated with this fibre bundle space. And $H l(\tilde{M})$ is indeed an extended ideal of $H(M)$.

In general, the group $H l(\tilde{M})$ may not form a Lie group ([3]). Yet, in many cases, $H l(\tilde{M})$ does form a Lie group. Assume that $H l(\tilde{M})$ is a Lie group. Let $H l^{\circ}(\tilde{M})$ denote the restricted Lie group of $H l(\tilde{M})$. In [3], the author studied the structure of the Lie algebra $d H l^{\circ}(\tilde{M})$ of $H l^{\circ}(\tilde{M})$. In this paper, we can use the results of [3] and go one step further to find an explicit expression of the structure of $d H l^{\circ}(\tilde{M})$. Then, we can easily show that the Nijenhuis's theorem is actually a very special case of the group $H l^{\circ}(\tilde{M})$.

For future use, we state Nijenhuis's theorem as follows:

"Let $h^{0}(M, p)$ be the restriced holonomy group. Then its Lie algebra $d h^{0}(M, p)$ is spanned by the matrices that arise from the $R_{i, \lambda}(x) ; x \in M$, by parallel transport to $p$ along any curves."

\section{§ 2. Priliminary.}

Let $(\tilde{M}, M, \pi)$ be a fibre bundle space. Assume that there is a 1-form $\omega$ on $\tilde{M}$ such that $\omega$ can determine the horizontal vectors at every point $P$ of $M$.

Received June 2, 1978. 
If a curve $C: I \rightarrow \tilde{M}, I$ being an interval, has horizontal tangents at all points, then $C$ is called a horizontal curve. Consider a curve $C: I \rightarrow M$ and let $C(0)=P_{0}$. Let $\widetilde{P}_{0}$ be a point in $\tilde{M}$ such that $\pi\left(\widetilde{P}_{0}\right)=P_{0}$. Suppose that there is a horizontal curve $\tilde{C}$ passing through $\widetilde{P}_{0}$ and $\pi(\widetilde{C})=C$. Then $\tilde{C}$ is unique and called the horizontal lift of $C$ passing through $\widetilde{P}_{0}$.

Now, let $r$ be a curve in $M$ joining two points $P_{0}$ and $P_{1}$ of $M$. Suppose that there is a horizontal lift $C$ of $r$. Then there are two neighborhoods $\bar{U}_{\lambda}$ of the fibre $F_{P_{\lambda}}$ containing $\tilde{P}_{\lambda}$, where $\lambda=0,1$, in such a way that for any point $Q_{0} \in F_{P_{0}}$ there is a unique horizontal curve passing through $Q_{0}$ and joining a point $Q_{1}$ in $\bar{U}_{1}$. Hence, we can define a mapping $\phi_{r}: \bar{U}_{0} \rightarrow \bar{U}_{1}$ by letting $\phi_{r}\left(Q_{0}\right)=Q_{1}$. Such a mapping is called a horizontal mapping covering $r$.

Now, take a closed curve $C$ from $x_{1}$ to $x_{1}$ in the base manifold $M$. The fibre over $x_{1}$ can be mapped onto itself by using horizontal mapping covering $C^{(*)}$. By considering all possible closed curves with finite arc length of $x_{1}$, a group of transformations on $F_{x_{1}}$ is obtained and we call this group the horizontal holonomy group. And we denote it by $\operatorname{Hl}\left(\tilde{M}, x_{1}\right)$.

Since we have that

$$
H l\left(M, x_{1}\right) \cong H l\left(M, x_{2}\right),
$$

for any two points $x_{1}$ and $x_{2}$ on $M$, we write $H l(\tilde{M})$ to denote the horizontal translation group which is attached to the space $\tilde{M}$.

First, we consider the horizontal holonomy group of a Riemannian fibred space. Let $(\tilde{M}, M, g, \pi)$ be a Riemannian fibred space. And the length $d s$ of a line segment in $\tilde{M}$ is given by

$$
d s^{2}=g_{j k}(y, x) d y^{j} d y^{k}+2 g_{j \alpha}(y, x) d y^{\jmath} d x^{\alpha}+g_{\alpha \beta}(y, x) d x^{\alpha} d x^{\beta},
$$

where the Greek letters $\alpha, \beta, \gamma$ etc. represent the coordinate system of the base manifold $M$ and the English letters $i, \jmath, k$ etc. represent the coordinate system of the fibre space. Define $\Gamma_{\alpha}^{i}$ as follows:

And also define

$$
\Gamma_{\alpha}^{i} g_{\imath \jmath}=g_{\jmath \alpha} \text {. }
$$

$$
K_{\alpha \beta}^{i}=\left(\partial_{\beta} \Gamma_{\alpha}^{i}-\partial_{\alpha} \Gamma_{\beta}^{i}\right)+\left(\Gamma_{\alpha}^{j} \partial_{j} \Gamma_{\beta}^{i}-\Gamma_{\beta}^{j} \partial_{\jmath} \Gamma_{\alpha}^{i}\right) .
$$

Then $K_{\alpha \beta}^{i}$ is a skew-symmetric tensor and $K_{\alpha \beta}=K_{\alpha_{\beta}}^{i} \partial_{2}$ is a infinitesimal vector field of a infinitesimal translation of $H l^{\circ}(\tilde{M})$ (see [3]).

Since $\Gamma_{\alpha}^{i}$ are functions of $\left(x^{\alpha}\right)$ and $\left(y^{2}\right)$, the vertical vector fields $K_{\beta \alpha}$ are functions of $\left(x^{\alpha}\right)$ and $\left(y^{i}\right)$. We denote them $K_{\beta a}(y, x)$. Let $P$ be a reference point on $M$. Then we have the followings:

Definition 1. We define the following set of vector fields on $F_{P}$.

$$
S=\left\{\bar{K}_{\beta \alpha}(y, x, r) \partial_{i} ; \alpha, \beta=1,2, \cdots, n, \quad \text { for all } x \text { and } r\right\},
$$

(*) In this paper, we assume that for any given curve $C$ on $M$, the horizontal liftings of $C$ always exist. The sufficient condition which makes the above statement true, has been discussed in [3]. 
where $\bar{K}_{\beta \alpha}^{\prime}(y, x, r) \partial_{\imath}$ are these vector fields obtained by translating $K_{\beta \alpha}(y, x)$ at the fibre $F_{x}$ to $F_{P}$ horizontally along any possible curve $r$ which connects the points $x$ and $P$.

Definition 2. Let $S$ be the set of vector fields defined in definition 1. If there exists a finite subset $\bar{S}=\left\{K_{1}, K_{2} \cdots K_{e}\right\}$ of $S$ such that

(1) Every element of $S$ is a linear combination of $\bar{S}$ over the real number.

(2) $\bar{S}$ forms a base of an involutive distribution, i. e. at every point $b$ of $F_{P},\left\{K_{1}(b), \cdots K_{e}(b)\right\}$ are linearly independent at $T_{P}$ and $\left[K_{\imath}, K_{\jmath}\right](b)$ belongs to the subspace generated by $\left\{K_{1}(b), \cdots, K_{e}(b)\right\}$.

In other words, $\bar{S}$ generates a submanifold of $F_{P}$ at every point $b$ of $F_{P}$. We say that $S$ is integrable.

Definition 3. Let $\phi_{r}$ be an element of $H l^{\circ}(\tilde{M}, P)$. A vector field $X \in F_{P}$ is said to be invariant under $\phi_{r}$ if

$$
\phi_{r_{*}} X=X
$$

A vector field $X \in F_{P}$ is called invariant under $H l^{\circ}(\tilde{M}, P)$ if it is invariant under all $\dot{\phi}_{r} \subseteq H l^{\circ}(\tilde{M}, P)$.

Definition 4. Let $X$ be a vector field on $F_{P}$. We say that $X$ is tangent to $H l^{\circ}(\tilde{M}, P)$ if (1) $X$ generates a global 1-parameter group $\phi$ and (2) $\phi_{t} \in H l^{\circ}(\tilde{M}, P)$ for all $t \in R$ (real number).

From [3], we have the following two theorems.

(A) If $H l^{\circ}(\tilde{M}, P)$ is a Lie group and $S$ is either integrable or invariant under $H l^{\circ}(\tilde{M}, P)$, then its Lie algebra $d H l^{\circ}(\tilde{M}, P)$ is spanned by $S$.

(B) Let $U$ be a neighborhood of $P$ and let all points and curves in the following arguments lie in $U$. $U$ may be chosen so that the local horizontal holonomy $\operatorname{group}^{(*)} H l^{*}(P)=H l^{\circ}(U . P)$. Let $\partial_{\alpha}, \alpha=1,2, \cdots, n$ be the coordinate vector fields of $U$. Construct the horizontal lifting $\partial_{\alpha}^{L}$ of $\partial_{\alpha}$. Let

$$
K=\left\{K_{\alpha \beta},\left[K_{\alpha \beta}, \partial_{\gamma}^{L}\right],\left[\left[K_{\alpha \beta}, \partial_{r}^{L}\right], \partial_{\partial}^{L}\right], \cdots\right\}(P)
$$

and $R(P)$ be the vector space spanned by $K$. Then we have that

Suppose that $\tilde{M}$ and $M$ are analytic Riemannian manifolds and $\Gamma_{\alpha}^{i}$ are analytic functions. If $H l^{*}(P)$ is a Lie group and $K$ is either integrable or invariant under $H l^{*}(P)$, then its Lie algebra $d H l^{*}(P)=R(P)$. Conversely, if $K$ is tangent to $H l^{*}(P)$, either integrable or invariant under $H l^{*}(P)$ and forming a finite Lie algebra, then $H l^{*}(P)$ is a Lie group.

\section{§3. Riemannian Fibred Space.}

Let $(\tilde{M}, M, g, \pi)$ be the Riemannian fibred space considered in section two. Define a vector field $V_{\alpha}$ in a coordinate neighborhood $U$ of $\tilde{M}$ as follows: Let

(*) The local horizontal holonomy group is defined in the same way as local linear holonomy group. 


$$
V_{\alpha}=\partial_{\alpha}-\Gamma_{\alpha}^{i} \partial_{i}
$$

Consider the inner product of $V_{\alpha}$ with the vertical vector field $\partial_{\jmath}$, 1. e. we have that

$$
\begin{aligned}
\left\langle V_{\alpha}, \partial_{\jmath}\right\rangle & =\left\langle\partial_{\alpha}, \partial_{\jmath}\right\rangle-\Gamma_{\alpha}^{i}\left\langle\partial_{i}, \partial_{\jmath}\right\rangle \\
& =g_{\alpha \jmath}-\Gamma_{\alpha}^{i} g_{\imath j}=g_{\alpha \jmath}-g_{\alpha \jmath}=0 .
\end{aligned}
$$

Hence, $V_{\alpha}$ is a horizontal vector field. Since we also have that $\pi_{*}\left(V_{\alpha}\right)=\partial_{\alpha}, V_{\alpha}$ is the horizontal lifting of $\partial_{\alpha}$. Now, let us calculate the Lie bracket of $V_{\alpha}$ and $V_{\beta}$. Then we obtain that

$$
\left[V_{\beta}, V_{\alpha}\right]=V_{\beta} V_{\alpha}-V_{\alpha} V_{\beta}=\partial_{\beta} \Gamma_{\alpha}^{\prime} \partial_{\imath}-\partial_{\alpha} \Gamma_{\beta}^{i} \partial_{i}-\Gamma_{\beta}^{\jmath} \partial_{j} \Gamma_{\alpha}^{\prime} \partial_{i}+\Gamma_{\alpha}^{j} \partial_{j} \Gamma_{\beta}^{i} \partial_{\imath}=K_{\alpha \beta} .
$$

Therefore, we obtain the following lemma:

LEMMA 1. Let $\partial_{\alpha}$ and $\partial_{\beta}$ be the coordinate vector fields of the base manfold $M$. Let $V_{\alpha}=\partial_{\alpha}-\Gamma_{\alpha}^{i} \partial_{i}$ and $V_{\beta}=\partial_{\beta}-\Gamma_{\beta}^{i} \partial_{2}$ be the horizontal lifting of $\partial_{\alpha}$ and $\partial_{\beta}$ respectively. Then

$$
K_{\alpha \beta}=\left[V_{\beta}, V_{\alpha}\right] \text {. }
$$

The Geometric Interpretation of $\left[V_{\beta}, V_{\alpha}\right]$.

First, let us look at a general case. Let $M$ be a differentiable manifold and $X$ and $Y$ be two vector fields on $M$. Refer to figure one. Let $C_{1}$ be the integral curve of $X$ from 0 to 1 with $C_{1}(t)=1, C_{2}$ be the integral curve of $Y$ from 1 to 2 with $C_{2}(t)=2, C_{3}$ be the integral curve of $X$ from 2 to 3 with $C_{3}(t)=3$ and $C_{4}$ be the integral curve of $Y$ from 3 to 4 with $C_{4}(t)=4$. By letting $t \rightarrow 0$, proved by Richard Faber, the tangent vector of the trace of point 4 reprsents the Lie bracket $[X, Y]$. Now, for the Lie bracket $\left[V_{\beta}, V_{\alpha}\right]$, we have a slightly different figure. Since $\left[\partial_{\beta}, \partial_{\alpha}\right]=0$, Refering to figure two, we can

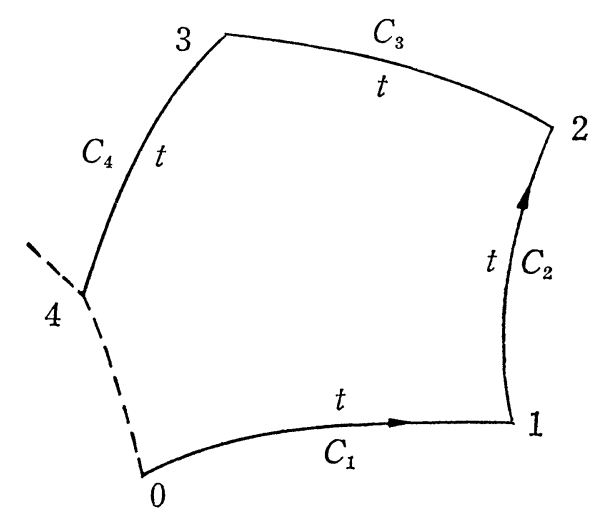

Fig. 1. 


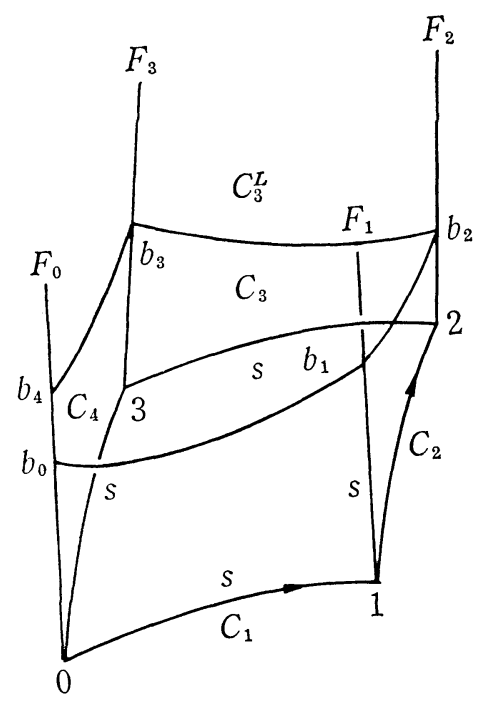

Fig. 2.

always find a closed curve $C_{1}+C_{2}+C_{3}+C_{4}$ on $M$ such that $C_{1}$ and $C_{3}$ are the integral curves of $\partial_{\beta}$ and $C_{2}$ and $C_{4}$ are the integral curves of $\partial_{\alpha}$. And all $C_{\imath}, \imath=1, \cdots, 4$, are of arc length $s$. Let $b_{0}$ be an arbitrary point on $F_{0}$. Consider the horizontal lifting $C_{\imath}^{L}, \imath=1, \cdots, 4$, of $C_{\imath}$. Then $C_{1}^{L}$ and $C_{3}^{L}$ are the integral curves of $V_{\beta}$ and $C_{2}^{L}$ and $C_{4}^{L}$ are the integral curves of $V_{\alpha}$. Hence, $\left[V_{\beta}, V_{\alpha}\right]$ represents the tangent vector of the trace of $b_{4}$. On the other hand, by letting $C=C_{1}+\cdots C_{4}, b_{4}$ represents the horizontal translation of $b_{0}$ to $b_{4}$ along the curve $C$ on $M$, i. e.

$$
b_{4}=\phi_{C}\left(b_{0}\right)
$$

Hence, by letting $s \rightarrow 0$, the tangent vector of the trace of $b_{4}$ is an infinitesimal horizontal translation of $H l^{\circ}(\tilde{M}, 0)$. This explains the geometric meaning of lemma 1.

\section{Fibre Bundle Space.}

By viewing the geometric meaning of $\left[V_{\beta}, V_{\alpha}\right]$, we know that the Riemannian metric did not play a important role. As long as the horizontal liftings of the coordinate vector fields are defined, the infinitesimal horizontal translations are defined. Besides, if for every $\partial_{\alpha}$ the horizontal lifting $V_{\alpha}$ is defined, then from equation 3.1, those quantities $\Gamma_{\alpha}^{i}$ are also defined. Hence, theorem (A) and (B) from Riemannian manifold, stated in section two, can be extended to any fibre bundle space. 
Now, we assume that $\tilde{M}$ is a fibre bundle space over $M$ such that the horizontal lifting of the coordinate vector fields are defined and differentiable. Then we obtain that

THEOREM 1. If $H l^{\circ}(\tilde{M}, P)$ is a Lie group and the following set $S^{\prime}$ is either integrable or invariant under $H l^{\circ}(\tilde{M}, P)$, then its Lie algebra is spanned by $S^{\prime}$, where

$$
\begin{gathered}
S^{\prime}=\left\{\phi_{r_{*}}\left[V_{\beta}, V_{\alpha}\right] ; \alpha, \beta=1,2, \cdots, n, r\right. \text { is any possible curve } \\
\text { on } M \text { which connects the points from } Q \text { to } P\}
\end{gathered}
$$

THEOREM 2. Suppose that $\tilde{M}$ and $M$ are analytus manifolds and the horizontal liftings of $\partial_{\alpha}, \alpha=1,2, \cdots, n$, are described by analytuc functions. If $H l^{*}(P)$ is a Lie group and the following set of vector fields $R(P)$ is either integrable or invariant under $H l^{*}(P)$, then its Lie algebra is spanned by $R(P)$, where

$$
R(P)=\left\{\left[V_{\beta}, V_{\alpha}\right],\left[\left[V_{\beta}, V_{\alpha}\right], V_{\gamma}\right], \cdots\right\} .
$$

Conversely, if $R(P)$ is tangent to $H l^{*}(P)$, either integrable or invariant under $H l^{*}(P)$ and forming a finte Lie algebra, then $H l^{*}(P)$ is a Lie group.

Applications :

(1) Let $\tilde{M}$ be the bundle of frames over $M$ and let the connection from $\omega$ be given. Then it is known that if $X$ and $Y$ are horizontal vector fields, then the vertical part of $[X, Y]$ is equal to $-2 \Omega(X, Y)([4]$, p. 35). Since the Lie bracket of $V_{\alpha}$ and $V_{\beta}$ is a vertical vector field, we have that

$$
\left[V_{\beta}, V_{\alpha}\right]=-2 \Omega\left(V_{\beta}, V_{\alpha}\right)=R\left(\partial_{\alpha}, \partial_{\beta}\right) \text {. }
$$

Also notice that the horizontal translation $\phi_{r}$ along a curve $r$, in this case, is the parallel translation along the curve $r$. Hence, theorem 1 says,

"The Lie algebra of the restricted holonomy group $H^{\circ}(\tilde{M}, P)$ is spanned by the set

$$
\left\{\tau_{r} \circ R\left(\partial_{\alpha}, \partial_{\beta}\right) \text {; for any possible curve } r \text { which connects } Q \text { and } P\right\} \text {. }
$$

This is exactly the same statement as Nijenhuis's theorem.

(2) Let $\tilde{M}$ be a fibre bundle space. We say that $\tilde{M}$ admits holonomy fibres, if at every point $P$ of $\tilde{M}$ there exists at least locally a submanifold of dimension $n$ orthogonal to the fibre passing through the point $P$.

THEOREM 3. Let $\tilde{M}$ be a fibre bundle space. $\tilde{M}$ admits holonomy fibres, if and only if $\left[V_{\beta}, V_{\alpha}\right]=0$, for all $\alpha, \beta=1,2, \cdots, n$.

Proof. The sufficient condition is obvious. If $\left[V_{\beta}, V_{\alpha}\right]=0$, then the horizontal distribution is involutive. Hence, it is integrable. For the necessary 
condition, since there exists a submanifold orthogonal to the fibre, $\left[V_{\beta}, V_{\alpha}\right]$ has to equal a horizontal vector. Yet, we know that $\left[V_{\beta}, V_{\alpha}\right]$ is a vertical vector. Hence it is equal to zero.\#

For a Riemannian fibred space, Muto proved the same theorem in [5].

(3) Let $\tilde{M}$ be a Riemannian fibred space. Since the torsion tensor $T$ is equal to zero, we have that

$$
\left[V_{\beta}, V_{\alpha}\right]=\tilde{\nabla}_{V_{\beta}} V_{\alpha}-\tilde{\nabla}_{V_{\alpha}} V_{\beta} .
$$

Hence, the Lie algebra of $H l^{\circ}(\tilde{M}, P$ ) (if it is a Lie group) is spanned by the vector fields of the above form.

(4) Let $\tilde{M}$ be a Riemannian fibred space with projectable metric. Then, $\left[V_{\beta}, V_{\alpha}\right]=2 h_{\beta}^{2} C_{\imath}=h\left(V_{\beta}, V_{\alpha}\right)([6])$, where $h$ is the second fundamental form of $\tilde{M}$ and $C_{\imath}$ are vertical vector fields. Hence, $d H l^{\circ}(\tilde{M}, P)$ is spanned by the vector fields $h\left(V_{\beta}, V_{\alpha}\right)$.

\section{The Associated Lie Algebra.}

In this section, we are looking for the connection between the de Rham's decomposition of a manifold and a special Lie algebra associated with the linear holonomy group.

Let $V R(P)$ be the vector space spanned by the set $R(P)$ in theorem 2 . Suppose that $V R(P)$ is a finite dimensional Lie algebra. By adding the set of vector fields $V_{\alpha} ; \alpha=1,2, \cdots, n$ to $V R(P)$, we obtain an enlarged vector space $V \bar{R}(P)$, i. e. $V \bar{R}(P)$ is spanned by the set

$$
\left\{V_{\alpha},\left[V_{\beta}, V_{\alpha}\right],\left[\left[V_{\beta}, V_{\alpha}\right], V_{\gamma}\right], \cdots\right\} \text {. }
$$

It is easy to show that $V \bar{R}(P)$ forms a finite Lie algebra and we call $V \bar{R}(P)$ the associated Lie algebra of $H l^{*}(U, P)$.

Now, let $M$ be an analytic manifold with an analytic connection. Assume that $M$ is a connected, simply connected and complete manifold. Then from [1] and theorem 2, $V R(P)$ is actually the linear holonomy group of $M$. Hence, $V \bar{R}(P)$ is the associated Lie algebra of $H(M)$.

In de Rham's theorem, let $T_{1}(P)$ and $T_{2}(P)$ be two orthogonal subspaces of the tangent space $T(P)$ and $T(P)=T_{1}(P)+T_{2}(P)$. Suppose that $T_{1}(P)$ (resp. $\left.T_{2}(P)\right)$ is invariant under the translation of the linear holonomy group of $M$. By parallel translating $T_{1}(P)$ (resp. $T_{2}(P)$ ) to all other points of $M$, then this vector distribution is integrable. Denote the integral manifold which passes through the point $P$ by $M_{1}$ (resp. $M_{2}$ ). Then de Rham's theorem says that ([7]).

" $M$ is isometric to the direct product $M_{1} \times M_{2}$." Let $V \bar{R}_{1}(P)\left(\right.$ resp. $\left.V \bar{R}_{2}(P)\right)$ be the associated Lie algebra of $H\left(M_{1}\right)$ (resp. $H\left(M_{2}\right)$ ). Then we have the following lemma :

LEMMA 2. $V \bar{R}_{1}(P)$ and $V \bar{R}_{2}(P)$ are zdeals of $V \bar{R}(P)$ and

$$
V \bar{R}(P)=V \bar{R}_{1}(P)+V \bar{R}_{2}(P) .
$$


Proof. Let $\partial_{\bar{\alpha}}, \bar{\alpha}=1,2, \cdots, m_{1}$ and $\partial_{\beta^{\prime}}, \beta^{\prime}=1,2, \cdots, m_{2}$ be the coordinate system of $M_{1}$ and $M_{2}$ respectively. Then

$$
\begin{aligned}
& V \bar{R}_{1}(P)=\text { the vector space spanned by }\left\{V_{\bar{\alpha}},\left[V_{\bar{\beta}}, V_{\bar{n}^{\prime}}, \cdots\right\},\right. \\
& V \bar{R}_{2}(P)=\text { the vector space spanned by }\left\{V_{\alpha^{\prime}},\left[V_{\beta^{\prime}}, V_{\alpha^{\prime}}\right], \cdots\right\} .
\end{aligned}
$$

From de Rham's theorem, we have that

$$
V \bar{R}(P)=V \bar{R}_{1}(P)+V \bar{R}_{2}(P) .
$$

Hence, we only have to show that $\mathrm{V} \bar{R}_{1}(P)$ (resp. $\mathrm{V} \bar{R}_{2}(P)$ ) is an ideal of $\mathrm{V} \bar{R}(P)$. It is the same to show that

$$
\left[\mathrm{V} \bar{R}_{1}(P), \mathrm{V} \bar{R}_{2}(P)\right]=0 .
$$

$$
\left[\mathrm{V}_{\bar{\alpha}}, \mathrm{V}_{\beta^{\prime}}\right]=0 .
$$

Referring to figure 3 , let $C_{1}$ and $C_{3}$ be the integral curves of $\partial_{\bar{\alpha}}$ and $\pi\left(C_{3}\right)=C_{1}$, i. e. $C_{3}$ and $C_{1}$ have the same $M_{1}$ coordinates. Let $C_{2}$ and $C_{4}$ be the integral curves of $\partial_{\beta^{\prime}}$ and $\pi\left(C_{2}\right)=C_{4}$. A vector $v_{2} \in T_{2}(P)$ is translated parallelly along the curve $C=C_{1}+C_{2}+C_{3}+C_{4}$. Then $v_{2}$ is invariant along $C_{1}$ and $C_{3}$ and equal along $C_{2}$ and $C_{4}$. Hence, $\tau_{C} v_{2}=v_{2}$. Similarly, for any $v_{1} \in T_{1}(P), \tau_{C} v_{1}=v_{1}$. Therefore, $\tau_{C} v=v$, for any $v \in T(P)$. By shrinking $C$ to zero, since $\left[V_{\bar{\alpha}}, V_{\beta^{\prime}}\right]$ represents the infinitesimal parallel translation of a vector along the curve $C$, we obtain that $\left[V_{\bar{\alpha}}, V_{\beta^{\prime}}\right]=0$.

$$
\left[\left[V_{\bar{\alpha}}, V_{\bar{\beta}}\right], V_{\gamma^{\prime}}\right]=0 .
$$

From Jacobi's identity, we have that

$$
\begin{aligned}
{\left[\left[V_{\bar{\alpha}}, V_{\bar{\beta}}\right], V_{\gamma^{\prime}}\right] } & =-\left[\left[V_{\gamma^{\prime}}, V_{\bar{\alpha}}\right], V_{\bar{\beta}}\right]-\left[\left[V_{\bar{\beta}}, V_{\gamma^{\prime}}\right], V_{\bar{\alpha}}\right] \\
& =0+0=0 .
\end{aligned}
$$

(3) By similar consideration, we obtain that

$$
\left[V \bar{R}_{1}(P), V_{\gamma^{\prime}}\right]=0 .
$$

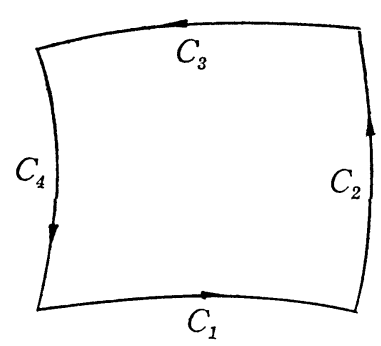

Fig. 3. 
Moreover, we have that

$$
\begin{aligned}
{\left[V \bar{R}_{1}(P),\left[V_{\beta^{\prime}}, V_{\gamma^{\prime}}\right]\right]=} & -\left[V_{\gamma^{\prime}},\left[V \bar{R}_{1}(P), V_{\beta^{\prime}}\right]\right] \\
& -\left[V_{\beta^{\prime}},\left[\mathrm{V}_{\gamma^{\prime}}, \mathrm{V} \bar{R}_{1}(P)\right]\right]=0 .
\end{aligned}
$$

(5) By induction, we can prove this lemma. \#

Hence, we conclude that

"The de Rham's decomposition of an analytic manifold, is associated with the decomposition of the Lie group $V R(P)$ into the direct sum of ideals."

\section{BIBLIOGRAPHY}

[1] A. Nijenituis, On the holonomy group of linear connection, Indagations 15 (1953), pp. 233-249.

[2] W. Ambrose and I. M. Singer, A theorem on holonomy, Trans. Amer. Math. Soc. Vol. 75 (1953) pp. 428-443.

[3] K. P. Cheng, Ph. D. thesis, McGill university, 1978.

[4] K. Nomizu, Lie group and differential geometry, the Math. Soc. of Japan, 1956.

[5] Y. Muto, On some properties of a fibred Riemannian manifold, Science report of the Yokohama National U. Sec. 1, No. 1, 1952.

[6] S. Ishinara and Konishi, Differential geometry and fibred space, Tokyo, 1973.

[7] S. Kobayasin and K. Nomizu, Foundations of Differential Geometry, Vol. 1, Interscience Publishers, 1963.

McGill University, Montreal, P.Q., Canada 